\title{
ChemComm
}

Cite this: Chem. Commun., 2013, 49, 10373

Received 26th May 2013, Accepted 13th June 2013

DOI: $10.1039 / c 3 c c 43955 k$

\section{Synthesis of a phosphine imide bearing a hydrosilane moiety, and its water-driven reduction to a phosphine†‡}

\author{
Naokazu Kano, ${ }^{\star}$ Kazuhide Yanaizumi, Xiangtai Meng, Nizam Havare and \\ Takayuki Kawashima*\&
}

www.rsc.org/chemcomm

Organosilanes bearing a phosphine imide moiety were synthesized and crystallographically characterized. Reaction of the pentacoordinated hydrodiphenylsilyl derivative with water gave [2-(diphenylphosphino)phenyl]diphenylsilanol accompanied by both reduction of the phosphine imide moiety and hydrolytic oxidation of the Si-H moiety.

Hydrosilanes, $\mathrm{R}_{3} \mathrm{Si}-\mathrm{H}$, are useful for hydrosilylation of multiple bonds, ${ }^{1}$ reduction of various functional groups, ${ }^{2}$ reductive aldol reactions, ${ }^{3}$ and preparation of a silanol, $\mathrm{R}_{3} \mathrm{Si}-\mathrm{OH}$, by hydrolysis. ${ }^{4}$ In some hydrosilanes, the silicon-bound hydrogen migrates intramolecularly through the 1,2-shift of the dyotropic rearrangement, ${ }^{5}$ 1,3-shift, ${ }^{6}$ and 1,5-shift. ${ }^{7}$ As represented by the 1,3-hydride shift, the hypercoordinated hydrosilanes show hydride reducing reactivity without catalysis. ${ }^{8}$ Some neutral pentacoordinated hydrosilanes react easily with alcohols, carboxylic acids, and water in the absence of a catalyst and evolve hydrogen gas, ${ }^{8 a, c, d}$ whereas tetracoordinated hydrosilanes react with them quite slowly even under harsh reaction conditions. $^{2}$ In these pentacoordinated hydrosilanes, the intramolecular coordination of a tertiary amino group with a suitable tether to the silicon atom is crucial for the pentacoordinated state of silicon and enhancement of the hydride reactivity. If a hydrosilyl group is located in the neighborhood of a phosphine imide, $\mathrm{R}_{3} \mathrm{P}=\mathrm{NR}^{\prime}$, the intramolecular $\mathrm{N} \cdots \mathrm{Si}$ coordination, which should increase its hydride reactivity, is expected. This expectation is based on the reports in which some phosphine imides have been used as a chelating ligand for several transition metal complexes. ${ }^{9}$ As far as we know, amongst the few reported silicon compounds bearing a phosphine imide ligand, no such hydrosilane has been reported. ${ }^{10}$ We report here the synthesis and structure of a hydrosilane bearing

Department of Chemistry, Graduate School of Science, The University of Tokyo, 7-3-1 Hongo, Bunkyo-ku, Tokyo 113-0033, Japan.E-mail: kano@chem.s.u-tokyo.ac.jp; Tel: +81358414338

$\dagger$ This paper is dedicated to Professor Renji Okazaki on the occasion of his 75th birthday.

‡ Electronic supplementary information (ESI) available: Experimental procedures and spectral data. CCDC 867751 (2a) and 867750 (2c). For ESI and crystallographic data in CIF or other electronic format see DOI: 10.1039/c3cc43955k $\S$ Present address: Graduate School of Science and Technology, Gunma University, 1-5-1 Tenjin-cho, Kiryu, Gunma 376-8515, Japan. a phosphine imide moiety and a unique conversion of its triarylphosphine imide moiety to the triarylphosphine moiety by hydride transfer from the hydrosilane moiety triggered by the reaction with water without any catalyst.

Phosphine imide 1 was lithiated with methyllithium in $\mathrm{Et}_{2} \mathrm{O}$ at room temperature, $, 11,12$ and then treated with chlorosilanes to give the corresponding silylated phosphine imides $2 \mathrm{a}-\mathbf{c}$ in 56, 59 and $64 \%$ yields, respectively, from 1 (Scheme 1). Compounds $2 a-c$ are thermally stable at room temperature in contrast to the phosphorus ylide analogue of 2a which was reported to undergo 1,4-silyl migration at $0{ }^{\circ} \mathrm{C}^{13}$ Compounds $\mathbf{2 a}$ and $\mathbf{2 b}$ are not sensitive to water. However, in contrast, compound $\mathbf{2 c}$ is very sensitive to moisture. Treatment of $2 \mathbf{c}$ with water at room temperature gave [2-(diphenylphosphino)phenyl]diphenylsilanol (3) quantitatively, based on the monitoring of the reaction using ${ }^{31} \mathrm{P}$ NMR spectroscopy and it was isolated in $87 \%$ yield (Scheme 2). The structure of 3 was determined using ${ }^{1} \mathrm{H},{ }^{13} \mathrm{C},{ }^{29} \mathrm{Si}$, and ${ }^{31} \mathrm{P}$ NMR spectroscopy, IR spectroscopy and mass spectrometry. The ${ }^{31} \mathrm{P}$ NMR spectrum $\left(\delta_{\mathrm{P}}-10.1 \mathrm{ppm}\right)$ established the existence of a triarylphosphine moiety in $\mathbf{3}$, instead of the corresponding triarylphosphine oxide moiety. Formation of the other product, tert-butylamine, which was derived from the imino moiety of 2c, was confirmed using GC-MS. Interestingly, the reaction of $2 \mathrm{c}$ with water resulted in the conversion from a hydrosilane and a triarylphosphine imide to a silanol and a set of a triarylphosphine and an amine, respectively. Therefore, this is not a usual hydrolysis of an iminophosphorane to give the corresponding phosphine oxide.

In order to reveal the causes of the reactivity of $2 \mathbf{c}$, we analyzed the structure in detail. The ${ }^{1} \mathrm{H},{ }^{13} \mathrm{C},{ }^{29} \mathrm{Si}$, and ${ }^{31} \mathrm{P} \mathrm{NMR}$

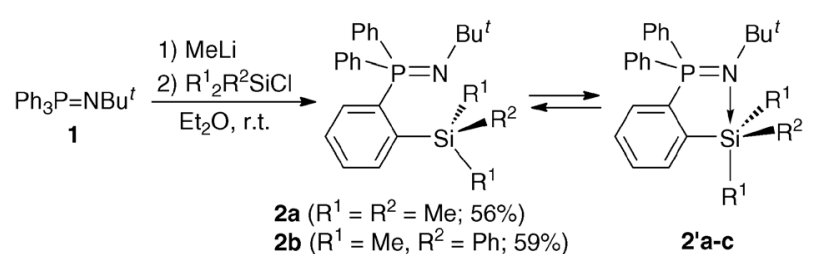

$2 b\left(R^{1}=M e, R^{2}=P h ; 59 \%\right)$

2'a-c

Scheme 1 


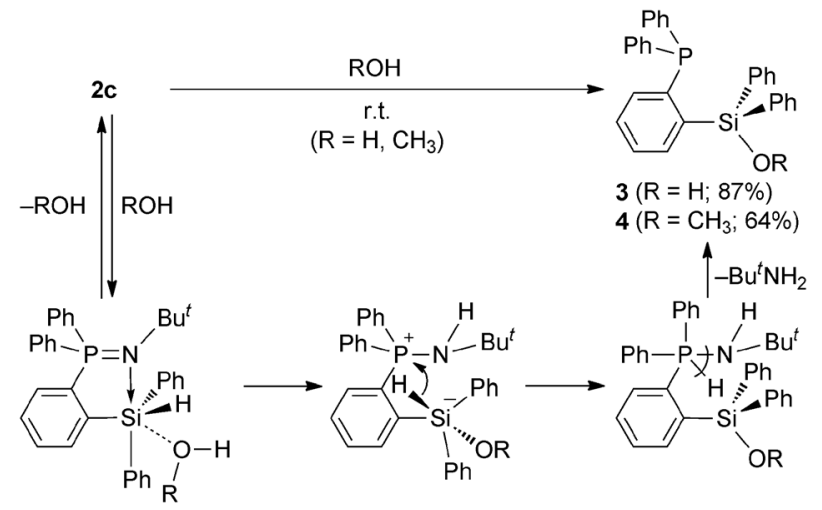

Scheme 2

spectroscopy results and elemental analysis of 2a-c were consistent with the silylated triarylphosphine imides. The ${ }^{29} \mathrm{Si}$ NMR spectra of $\mathbf{2 a}$ and $\mathbf{2 b}$ in $\mathrm{CDCl}_{3}$ showed only small downfield shifts (2a: $\delta_{\mathrm{Si}}-2.8 \mathrm{ppm} ; 2 \mathbf{b}: \delta_{\mathrm{Si}}-5.2 \mathrm{ppm}$ ) compared to trimethylphenylsilane $\left(\delta_{\mathrm{Si}}-4.1 \mathrm{ppm}\right)^{14}$ and dimethyldiphenylsilane $\left(\delta_{\mathrm{Si}}-8.6 \mathrm{ppm}\right),{ }^{15}$ respectively. In contrast, $2 \mathrm{c}$ showed a significant upfield shift and an increased $\mathrm{Si}-\mathrm{H}$ coupling constant $\left(\delta_{\mathrm{Si}}-26.4 \mathrm{ppm}\left(\mathrm{d},{ }^{1} J_{\mathrm{SiH}}=231 \mathrm{~Hz}\right)\right)$ compared to triphenylsilane $\left(\delta_{\mathrm{Si}}-17.4 \mathrm{ppm}\left(\mathrm{d},{ }^{1} J_{\mathrm{SiH}}=199 \mathrm{~Hz}\right)\right)$ in $\mathrm{C}_{6} \mathrm{D}_{6}$ at room temperature. In the VT-NMR experiment, lowering the temperature of a THF- $d_{8}$ solution of $2 \mathrm{c}$ caused an increased upfield shift $\left[\delta_{\mathrm{Si}}-25.6 \mathrm{ppm}\right.$ at $25{ }^{\circ} \mathrm{C}, \delta_{\mathrm{Si}}-29.7 \mathrm{ppm}$ at $-60{ }^{\circ} \mathrm{C}$. These results indicate the existence of a dynamic equilibrium between the chelated pentacoordinated and unchelated tetracoordinated silicon states in solution as shown in Scheme 1 and, especially at low temperature, a certain degree of contribution of the pentacoordination state represented by $2^{\prime} \mathbf{c} .^{16}$

To confirm the contribution of a pentacoordination state of silicon in $\mathbf{2 c}$, the crystal structures of trimethylsilane $2 \mathbf{a}$ and hydrodiphenylsilane 2c were determined by X-ray crystallographic analysis (Fig. 1). The N1 atom is located on the backside of the Si1-C23 bond and directed to the silyl group in both 2a

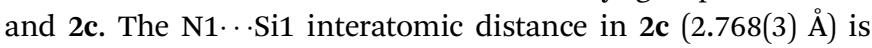
much shorter than the corresponding distance in 2a (3.353(1) $\mathrm{A})$ and the sum of the van der Waals radii (3.54 $\AA$ ). The bond angle $\angle \mathrm{C} 1-\mathrm{Si} 1-\mathrm{C} 23$ in 2c $\left(104.05(12)^{\circ}\right)$ is somewhat narrower than that $\left(107.08(6)^{\circ}\right)$ in $2 a$. These structural features show that $2 \mathbf{c}$ is a capped tetrahedral structure around the pentacoordinated Si1 atom. The difference in the degree of the intramolecular $\mathrm{N} \cdots \mathrm{Si}$ coordination between $2 \mathbf{a}$ and $2 \mathbf{c}$ is ascribed to the difference in the electrophilicity of silicon. Considering that the hydrodiphenylsilane bearing 2-(phenylazo)phenyl group provides no N. SSi interaction, ${ }^{17}$ the phosphine imide acts as a good coordinating ligand to achieve the pentacoordinated silicon state, reflecting the polarized $\mathrm{P}-\mathrm{N}$ bond character. ${ }^{9 b}$

Based on the contribution of the pentacoordinated silicon in $\mathbf{2 c}$, a mechanism of formation for $\mathbf{3}$ was proposed as shown in Scheme 2. Formation of $\mathbf{3}$ from $\mathbf{2 c}$ is initiated by reversible coordination of water to the silicon atom, whose Lewis acidity is enhanced by the contribution of the pentacoordinated state based on the intramolecular $\mathrm{N} \cdots \mathrm{Si}$ coordination in $\mathbf{2 c},{ }^{18}$ and proton transfer to the neighboring nitrogen forms a silicate intermediate.

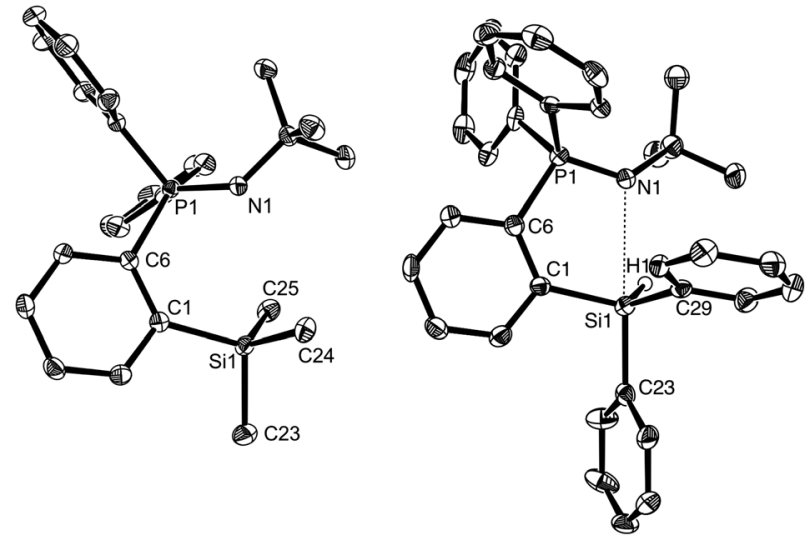

Fig. 1 ORTEP drawings of $2 \mathrm{a}$ (left) and $\mathbf{2 c}$ (right) with the thermal ellipsoid plot (50\% probability). Hydrogen atoms except for $\mathrm{H} 1$ in $2 \mathrm{c}$ are omitted for clarity. Selected bond lengths $[\AA]$ and bond angles $\left[{ }^{\circ}\right]$ of 2a: Si1-C1 1.9043(12), Si1-C23 1.8796(14), Si1-C24 1.8617(13), Si1-C25 1.8693(13), Si1 ..N1 3.353(1), N1-P1 1.5479(10), C1-Si1-C23 107.08(6), C1-Si1-C24 110.22(6), C1-Si1-C25 114.01(5), C23-Si1-C24 106.51(6), C23-Si1-C25 105.50(6), C24-Si1-C25 112.94(6). 2c: Si1-C1 1.900(3), Si1-C23 1.905(3), Si1-C29 1.880(3), Si1-H1 1.43(2), Si1...N1 2.768(3), N1-P1 1.561(2), C1-Si1-C23 104.05(12), C1-Si1-C29 111.97(12), C23-Si1-C29 104.65(13), C1-Si1-H1 114.6(9), C23-Si1-H1 105.6(9), C29-Si1-H1 114.7(9).

Hydride transfer from silicon to phosphorus generates an amino(hydro)phosphorane intermediate, and its facile reductive deamination gives 3 and tert-butylamine as the final products. In another possible reaction mechanism, the phosphine imide moiety, which may work as a base, is hydrolyzed to a phosphine oxide and tert-butylamine before the conversion of the hydrosilane moiety to silanol. We carried out three experiments to determine the reaction mechanism: (i) monitoring of the reaction solution using VT-NMR spectroscopy, (ii) a labeling experiment using $\mathrm{D}_{2} \mathrm{O}$ instead of $\mathrm{H}_{2} \mathrm{O}$, and (iii) a reaction of 2c with methanol. In experiment (i), however, the formation of 3 from $2 \mathrm{c}$ was so fast that no intermediate could be observed using ${ }^{1} \mathrm{H}$ and ${ }^{31} \mathrm{P}$ NMR spectroscopy even at $-20{ }^{\circ} \mathrm{C}$. In experiment (ii), quantitative formation of $2-\left(\mathrm{Ph}_{2} \mathrm{P}\right) \mathrm{C}_{6} \mathrm{H}_{4} \mathrm{Si}(\mathrm{OD}) \mathrm{Ph}_{2}$ (3-d) and $\mathrm{Bu}^{t} \mathrm{NHD}$ in the reaction of $2 \mathrm{c}$ with $\mathrm{D}_{2} \mathrm{O}$ was confirmed using ${ }^{1} \mathrm{H}$ and ${ }^{31} \mathrm{P}$ NMR spectroscopy. The GC-MS measurement also supported the formation of $\mathrm{Bu}^{t} \mathrm{NHD}\left(\mathrm{m} / z=74, \mathbf{M}^{+}\right)$. Therefore, silicon-bound hydrogen in $2 \mathbf{c}$ was used for the quantitative formation of $\mathrm{Bu}^{t} \mathrm{NH}_{2}$ but not used for the formation of hydrogen gas in the reaction with water. The experimental result is consistent with the proposed reaction mechanism in Scheme 2. Combination of the characteristics of both pentacoordinated silicon and phosphorus should be a key for this reaction of 2c. In experiment (iii), the reaction of 2c with methanol gave methoxysilane 4 in $64 \%$ yield (Scheme 2). Therefore, even though $\mathrm{OH}^{-}$or $\mathrm{MeO}^{-}$may attack the phosphorus in any of the steps, the intermediate should give 2c back and finally give 3 or 4 . Experiments (ii) and (iii) rule out other reaction mechanisms involving the hydrolysis of the phosphine imide moiety.

In the previous reports, phosphine imides were reduced with strong reductants such as lithium aluminum hydride and trichlorosilane, which react vigorously with water. ${ }^{19}$ In contrast, the reduction of the phosphine imide of $\mathbf{2 c}$ to phosphine was promoted by 
the reaction with water and it proceeded quickly at room temperature. Simultaneously, the hydrosilane moiety was converted to the silanol moiety. In association with this reactivity of $2 \mathrm{c}$ to give 3 and 4, dehydrogenative condensation of hydrosilanes with alcohols or amines without additional catalyst has been reported by combination of a triarylborane moiety in a single molecule, although condensation with water was not mentioned. ${ }^{20}$ In addition, some hypercoordinated silanes evolve hydrogen gas in the condensation with alcohols, carboxylic acids and water. ${ }^{8 a, c, d}$ Therefore, it is notable that the conversion of $\mathbf{2 c}$ to $\mathbf{3}$ and $\mathbf{4}$ proceeds at room temperature without any catalyst evolving hydrogen gas.

In summary, three organosilicon compounds $\mathbf{2 a - c}$ bearing a phosphine imide moiety were synthesized. The hydrosilane moiety of the hydrodiphenylsilyl derivative $2 \mathrm{c}$ was hydrolyzed without any catalyst to the corresponding silanol accompanied by the reduction of the iminophosphorane moiety to triarylphosphine. Its high reactivity can be ascribed to the characteristics of both highly coordinated silicon and phosphorus in the intermediates. The unique structure of the product, which bears both the silanol and the triarylphosphine moiety at the ortho-position, is expected to be useful as a precursor of an $O, P$-chelating ligand for transition metal complexes. ${ }^{21}$

We thank Tosoh Finechem Corp. and Shin-etsu Chemical Co. Ltd. for gifts of alkyllithiums and silicon reagents, respectively. This work was partially supported by Grants-in-Aid for the Global COE Program for Chemistry Innovation and for Scientific Research (No. 22108508, 22685005 and 25109512) from the Ministry of Education, Culture, Sports, Science and Technology, Japan.

\section{Notes and references}

1 (a) B. Marciniec, J. Gulinski, W. Urbaniak and Z. W. Kornetka, in Comprehensive Handbook on Hydrosilylation, ed. B. Marciniec, Pergamon Press, Oxford, United Kingdom, 1992; (b) B. Marciniec, H. Maciejewski, C. Pietraszuk and P. Pawluc, in Hydrosilylation. A Comprehensive Review on Recent Advances, ed. B. Marciniec, Springer, 2009; (c) D. Addis, S. Das, K. Junge and M. Beller, Angew. Chem., Int. Ed., 2011, 50, 6004.

2 M. A. Brook, Silicon in Organic, Organometallic, and Polymer Chemistry, Wiley, New York, 2000, ch. 8, pp. 171-188.

3 H. Nishiyama and T. Shiomi, Top. Curr. Chem., 2007, 279, 105.

4 P. D. Lickiss, Adv. Inorg. Chem., 1995, 42, 147.

5 J. Fassler and S. Bienz, Organometallics, 1994, 13, 4704.

6 (a) R. J. P. Corriu, G. F. Lanneau and M. Perrot, Tetrahedron Lett., 1987, 28, 3941; (b) M. Yamamura, N. Kano and T. Kawashima, Tetrahedron Lett., 2007, 48, 4033; (c) E. Kertsnus-Banchik, I. Kalikhman, B. Gostevskii, Z. Deutsch, M. Botoshansky and D. Kost, Organometallics, 2008, 27, 5285; (d) K. Lippe, D. Gerlach, E. Kroke and J. Wagler, Organometallics, 2009, 28, 621; (e) M. Yamamura, N. Kano and T. Kawashima, Z. Anorg. Allg. Chem., 2009, 635, 1295.
7 (a) S. Anwar and A. P. Davis, J. Chem. Soc., Chem. Commun., 1986, 831; (b) S. W. McCombie, C. Ortiz, B. Cox and A. K. Ganguly, Synlett, 1993, 541.

8 (a) J. Boyer, C. Breliere, R. J. P. Corriu, A. Kpoton, M. Poirier and G. Royo, J. Organomet. Chem., 1986, 311, C39; (b) R. J. P. Corriu, G. F. Lanneau and M. Perrot, Tetrahedron Lett., 1988, 29, 1271; (c) P. Arya, R. J. P. Corriu, K. Gupta, G. F. Lanneau and Z. Yu, J. Organomet. Chem., 1990, 399, 11; (d) R. J. P. Corriu, G. F. Lanneau, M. Perrot-Petta and V. D. Nehta, Tetrahedron Lett., 1990, 31, 2585; (e) R. J. P. Corriu, G. F. Lanneau and Z. Yu, Tetrahedron, 1993, 49, 9019; $(f)$ Y. Domoto, A. Fukushima, Y. Kasuga, S. Sase, K. Goto and T. Kawashima, Org. Lett., 2010, 12, 2586.

9 (a) S. Wingerter, H. Gornitzka, G. Bertrand and D. Stalke, Eur. J. Inorg. Chem., 1999, 173; (b) A. Steiner, S. Zacchini and P. I. Richards, Coord. Chem. Rev., 2002, 227, 193; (c) J. Vicente, J.-A. Abad, R. Clemente, J. Lopéz-Serrano, M. C. Ramírez de Arellano, P. G. Jones and D. Bautista, Organometallics, 2003, 22, 4248; (d) R. Bielsa, A. Larrea, R. Navarro, T. Soler and E. P. Urriolabeitia, Eur. J. Inorg. Chem., 2005, 1724; (e) D. Aguilar, M. Contel, R. Navarro and E. P. Urriolabeitia, Organometallics, 2007, 26, 4604; $(f)$ R. Bielsa, R. Navarro, E. P. Urriolabeitia and A. Lledós, Inorg. Chem., 2007, 46, 10133; $(g)$ E. Martinez-Arripe, F. Jean-Baptiste-dit-Dominique, A. Auffrant, X.-F. LeGoff, J. Thuilliez and F. Nief, Organometallics, 2012, 31, 4854.

10 (a) W. Wolfberger, H.-H. Pickel and H. Schnzidbaur, Chem. Ber., 1971, 104, 1830; (b) A. Sivaramakrishna, I. Kalikhman, E. Kertsnus, A. A. Korlyukov and D. Kost, Organometallics, 2006, 25, 3665; (c) Y. Xiong, S. Yao, S. Inoue, E. Irran and M. Driess, Angew. Chem., Int. Ed., 2012, 51, 10074.

11 (a) P. Wei, K. T. K. Chan and D. W. Stephan, Dalton Trans., 2003, 3804; (b) K. T. K. Chan, L. P. Spencer, J. D. Masuda, J. S. J. McCahill, P. Wei and D. W. Stephan, Organometallics, 2004, 23, 381; (c) L. Boubekeur, L. Ricard, N. Mézailles, M. Demange, A. Auffrant and P. Le Floch, Organometallics, 2006, 25, 3091.

12 N. Kano, K. Yanaizumi, X. Meng and T. Kawashima, Heteroat. Chem., 2012, 23, 429.

13 A. Kawachi, T. Yoshioka and Y. Yamamoto, Organometallics, 2006, 25, 2390.

14 C. Breliére, F. Carré, R. J. P. Corriu, M. Poirier and G. Royo, Organometallics, 1986, 5, 388.

15 R. H. Cragg and R. D. Lane, J. Organomet. Chem., 1984, 277, 199.

16 (a) B. J. Helmer, R. West, R. J. P. Corriu, M. Poirier, G. Royo and A. De Saxce, J. Organomet. Chem., 1983, 251, 295; (b) N. Auner, R. Probst, F. Hahn and E. Herdtweck, J. Organomet. Chem., 1993, 459, 25.

17 (a) N. Kano, F. Komatsu and T. Kawashima, Chem. Lett., 2001, 338; (b) N. Kano, F. Komatsu, M. Yamamura and T. Kawashima, J. Am. Chem. Soc., 2006, 128, 7097.

18 M. A. Brook, Silicon in Organic, Organometallic, and Polymer Chemistry, Wiley, New York, 2000, chap. 4, pp. 97-114.

19 (a) L. D. Quin, G. Keglevich and K. C. Caster, Phosphorus, Sulfur Relat. Elem., 1987, 31, 133; (b) J. Barluenga, F. López and F. Palacios, Tetrahedron Lett., 1987, 28, 2875; (c) J. Barluenga, F. López and F. Palacios, Synthesis, 1989, 298.

20 (a) A. Kawachi, M. Zaima, A. Tani and Y. Yamamoto, Chem. Lett., 2007, 362; (b) L. Könczöl, A. Kawachi and D. Szieberth, Organometallics, 2012, 31, 120.

21 (a) P. W. N. M. van Leeuwen, C. F. Roobeek and A. G. Orpen, Organometallics, 1990, 9, 2179; (b) M. A. Garralda, R. Hernández, L. Ibarlucea, E. Pinilla, M. R. Torres and M. Zarandona, Organometallics, 2007, 26, 1031; (c) N. Yoshikai, H. Matsuda and E. Nakamura, J. Am. Chem. Soc., 2009, 131, 9590. 